\title{
Initiation of ventricular fibrillation outside hospital in patients with acute ischaemic heart disease
}

\author{
A A J ADGEY, J E DEVLIN, $S$ W WEBB, H C MULHOLLAND \\ From the Regional Medical Cardiology Centre, Royal Victoria Hospital, Grosvenor Road, Belfast, Northern Ireland
}

SUMMARY Factors which may predispose to ventricular fibrillation were assessed in $\mathbf{4 8}$ consecutive patients who developed ventricular fibrillation outside hospital after the arrival of a mobile coronary care team. Before the initiation of ventricular fibrillation, late cýcle ventricular extrasystoles were recorded in 38 patients (79\%) and $R$ on $T$ extrasystoles in 27 patients (56\%). R on $\mathrm{T}$ extrasystoles were relatively infrequent-an average of three occurred during a mean monitoring time of 27 minutes. Multifocal extrasystoles occurred in only three patients $(6 \%)$, consecutive extrasystoles in $14(29 \%)$, and self-terminating ventricular tachycardia in three $(6 \%)$. Ventricular extrasystoles occurring at more than five a minute were uncommon during this phase. The time from "warning arrhythmias" to the development of ventricular fibrillation in many patients was short, thus limiting the administration of antiarrhythmic agents. There was a significant increase in the heart rates recorded immediately before ventricular fibrillation when compared with those documented initially. Thus, an increase in heart rate appeared to be a predisposing factor in the initiation of ventricular fibrillation.

An $\mathrm{R}$ on $\mathrm{T}$ extrasystole was the most important factor in the initiation of ventricular fibrillation: it occurred in 33 patients (69\%). In nine (19\%) ventricular tachycardia and in six (12\%) a late cycle extrasystole or idioventricular rhythm initiated ventricular fibrillation.

During the early minutes or hours after the onset of myocardial ischaemia or infarction, ventricular fibrillation is the major complication. The majority of patients are in ventricular fibrillation when first seen by prehospital units. Thus, it has been found ${ }^{1}$ that among patients with primary ventricular fibrillation (fibrillation in the absence of pump failure or cardiogenic shock) during the first hour of myocardial infarction, $82 \%$ were in ventricular fibrillation before the mobile team arrived. Nevertheless, in order to assess factors that may initiate ventricular fibrillation, we decided to review the data on patients who developed it outside hospital after the arrival of the Belfast Mobile Coronary Care Unit.

\section{Patients and methods}

Between October 1972 and April 1977 we studied consecutively patients who developed ventricular fibrillation outside hospital. On arrival of the prehospital unit, the patient was immediately connected to

Accepted for publication 2 September 1981 an oscilloscope, and a two channel tape recorder. One channel allowed continuous recording of the electrocardiogram, the other, a speech channel, permitted the frequent documentation of blood pressure recordings by sphygmomanometer, and the time at which drugs were administered. A "Venflon" cannula was inserted into a forearm vein for the intravenous administration of drugs.

Treatment given by the family doctor, for example analgesics, atropine, or lignocaine, was recorded. We excluded from the study patients with ventricular fibrillation or sustained ventricular tachycardia when first seen.

Electrocardiographic monitoring was continuous during transport and for 10 minutes after the patient arrived in the hospital coronary care unit. Blood pressure was recorded on arrival with the patient, and at intervals before the patient was moved.

A permanent record of the electrocardiogram was obtained from tape recordings, and several specific heart rates were documented: (1) The heart rate when the patient was first seen and connected to the tape recorder (initial). (2) The heart rate immediately pre- 
ceding ventricular fibrillation (preventricular fibrillation). (3) The heart rate immediately before transfer in patients who developed ventricular fibrillation while being moved, or in the ambulance during the journey to hospital (premovement). The cardiac rhythm, frequency and type of extrasystoles, blood pressure, and therapeutic interventions were analysed from the tape recordings, and the results subjected to statistical analysis.

The diagnosis of myocardial infarction was made when at least two of the following were present: typical chest pain, clinically significant rise in cardiac enzymes, or characteristic evolving changes on the electrocardiogram. Myocardial infarction was also diagnosed at necropsy. An acute ischaemic episode was defined as prolonged chest pain at rest caused by acute myocardial ischaemia without evidence of infarction.

The prematurity index of ventricular premature beats was assessed by dividing the coupling interval of the ventricular premature beat $\left(R R^{\prime}\right)$ by the $Q T$ interval of the preceding normally conducted beat. A ventricular extrasystole with a prematurity index of $<1$ was considered to represent the $R$ on $T$ phenomenon, while a premature beat with an index of $\geqslant 1$ was termed long-coupling or late cycle.

\section{Results}

Fifty-two patients entered the study. Three patients were excluded because of malfunction of the recording apparatus and the record was unobtainable for analysis in a further patient. The study, therefore, was made up of 48 patients. Forty were men, aged 37 to 79 years (mean age 56 years), and eight were women aged 40 to 76 (mean age 59 years). The site of infarction was anterior in $29(63 \%)$, inferior in 15 (33\%), anterior and inferior in one, and subendocardial in one. One patient had myocardial ischaemia, and aortic stenosis was present in the remaining patient. Thirteen patients had had one or more previous myocardial infarctions. Thirty-nine patients had primary ventricular fibrillation and nine had secondary ventricular fibrillation.

The median time from onset of symptoms of myocardial infarction to arrival of the mobile coronary care unit was 68 minutes. Twenty-two patients were seen within one hour. The median time from onset of symptoms to development of ventricular fibrillation was 105 minutes. Ten patients developed ventricular fibrillation within one hour.

The electrocardiographic monitoring time before development of ventricular fibrillation varied from one to 80 minutes (mean 25 minutes).

Routine treatment before the acute incident consisted of beta-blocking agents in three patients, methyldopa in two, guanethidine in one, and glycer trinitrate in one patient. Before ventricular fibrillstion, 37 patients received analgesics either from the family practitioner or from members of the mobife coronary care unit. Antiarrhythmic, vagolytic, anc beta-sympatholytic agents administered intravenous and before ventricular fibrillation were atropin (eight), lignocaine (three), sotalol (two), atropine and practolol (atropine $0.6 \mathrm{mg}$, practolol $10 \mathrm{mg}$ ) (twoos and atropine and sotalol (atropine $0.6 \mathrm{mg}$, sotalol 8 $\mathrm{mg}$ ) (two). Atter the administration of atropine or beta-blocking agents no patient developed tachya rhythmia with or without hypertension or bradyas rhythmia with or without hypotension.

On arrival of the mobile team, 25 patients were sinus rhythm, 10 had sinus tachycardia, 10 had sinus bradycardia, two had atrial fibrillation, and one haet second degree atrioventricular block. The blood pres sure was recorded on arrival in 42 patients. In six patients it was not possible to take a blood pressure recording since the speed of development of ventricit lar fibrillation precluded the estimation of the blop pressure. The range of systolic pressures was 60 to $195 \mathrm{mmHg}$ (mean $124 \mathrm{mmHg}$ ). The rhythm and blood pressure recorded immediately before the initiation of ventricular fibrillation showed that 28 patients were in sinus rhythm, 12 had sinus tachycas dia, and two sinus bradycardia. Five had atrial fibrid lation. Idioventricular rhythm was present in one and ventricular tachycardia in one. Both were sei terminating. Blood pressure was recorded in 41 of the 48 patients; the systolic blood pressure varied from $\bar{B}$ to $200 \mathrm{mmHg}$ (mean $125 \mathrm{mmHg}$ ).

The range of the initial heart rates and those before ventricular fibrillation are recorded in Table 1 . There was a significant increase in the heart rates immedo ately before ventricular fibrillation (paired $t$ test of the differences between the initial and preventricular. fibrillation heart rates, $p<0.001$ ). On excluding those patients who had received atropine or a beta-blocking agent, the $p$ value was still significant $(p<0.015$. Fig. 1 shows the distribution of the initial and preventricular fibrillation heart rates. An increase in heat rate is shown for the majority of patients. In 12 of the 48 patients, ventricular fibrillation occurred during movement of the patient from the position when finst seen (Table 2). There was a significant increase bet ween the initial and premovement heart rates age

Table 1 Initial and preventricular fibrillation heart rates in 48 patients

\begin{tabular}{|c|c|}
\hline $\begin{array}{l}\text { Initial } \\
\text { Pre-VF }\end{array}$ & $\begin{array}{l}47-175(\text { mean } 84 \pm 4.14 \mathrm{SEM}) / \mathrm{min} \\
52-214(\text { mean } 98 \pm 4.99 \mathrm{SEM}) / \mathrm{min}\end{array}$ \\
\hline \multicolumn{2}{|c|}{ Paired $\mathrm{t}$ test $\mathrm{p}<0.001$} \\
\hline
\end{tabular}




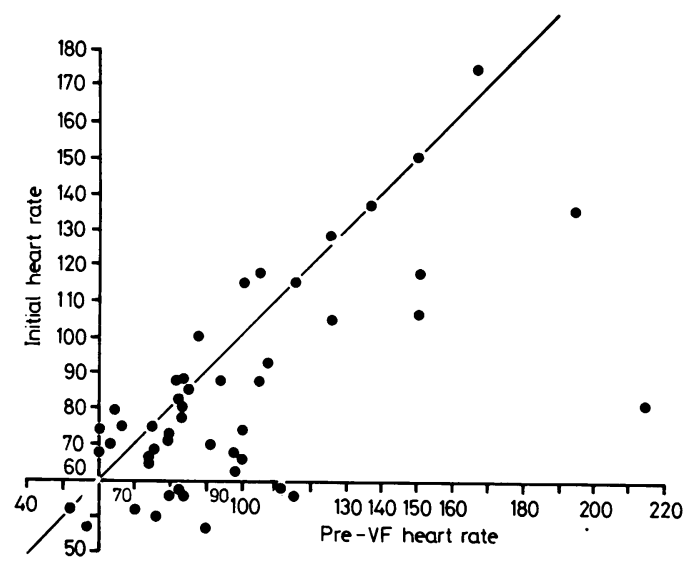

Fig. 1 Initial and preventricular fibrillation heart rates in the 48 patients. The line drawn represents a heart rate of no change.

between the initial and preventricular fibrillation heart rates.

Extrasystolic activity preceding ventricular fibrillation but excluding that initiating ventricular fibrillation was recorded (Table 3). Seventy-nine per cent had late cycle extrasystoles and $56 \%$ had $\mathrm{R}$ on $\mathrm{T}$ extrasystoles. Consecutive, multifocal, bigeminal ventricular extrasystoles, self-terminating ventricular tachycardia, and atrial extrasystoles were relatively

Table 2 Initial, premovement and preventricular fibrillation heart rates in 12 patients where ventricular fibrillation occurred during movement

\begin{tabular}{lll}
\hline (A) & Initial heart rate & $48-136$ (mean $85 \pm 8.96 \mathrm{SEM}) / \mathrm{min}$ \\
(B) & Premovement heart rate & $48-152($ mean $95.5 \pm 9.96 \mathrm{SEM}) / \mathrm{min}$ \\
(C) & Pre-VF heart rate & $56-194$ (mean $104 \pm 11.06 \mathrm{SEM} / \mathrm{min}$
\end{tabular}

Paired $t$ test between (A) and (B) $0.05>p>0.025$.

Paired $t$ test between (A) and (C) $0.02>p>0.01$.

Table 3 Extrasystoles recorded before initiation of ventricular fibrillation

\begin{tabular}{lll}
\hline & $\begin{array}{l}\text { No. of } \\
\text { patients }\end{array}$ & $\begin{array}{l}\text { Incidence } \\
\%\end{array}$ \\
\hline Late cycle ventricular extrasystoles & 38 & 79 \\
R on T ventricular extrasystoles & 27 & 56 \\
Consecutive ventricular extrasystoles & 14 & 29 \\
Multifocal ventricular extrasystoles & 3 & 6 \\
Bigeminal ventricular extrasystoles & 3 & 6 \\
VT (self-terminating) & 3 & 6 \\
Atrial extrasystoles & 3 & 6 \\
No extrasystolic activity & 6 & 12 \\
\hline
\end{tabular}

Consecutive ventricular extrasystoles-two successive ventricular extrasystoles occurring in pairs. Bigeminal ventricular

extrasystoles-a ventricular extrasystole alternating with a normally conducted beat for at least two normally conducted beats. VT

(self-terminating) ventricular tachycardia-three or more successive ventricular extrasystoles at a ventricular rate of $>120$ /minute and self-terminating. infrequent. In six patients no ventricular extrasystoles were recorded. They had a shorter average monitoring time (nine minutes) than that of the whole group. The frequency distribution of late cycle ventricular extrasystoles in relation to the monitoring time is shown in Fig. 2. The average number of late cycle ventricular extrasystoles recorded was 13 for a mean monitoring time of 27 minutes. A similar graph (Fig. 3) plotted the frequency distribution of $R$ on $T$ extrasystoles and showed an average of three $R$ on $T$ extrasystoles for a mean monitoring time of 27 minutes.

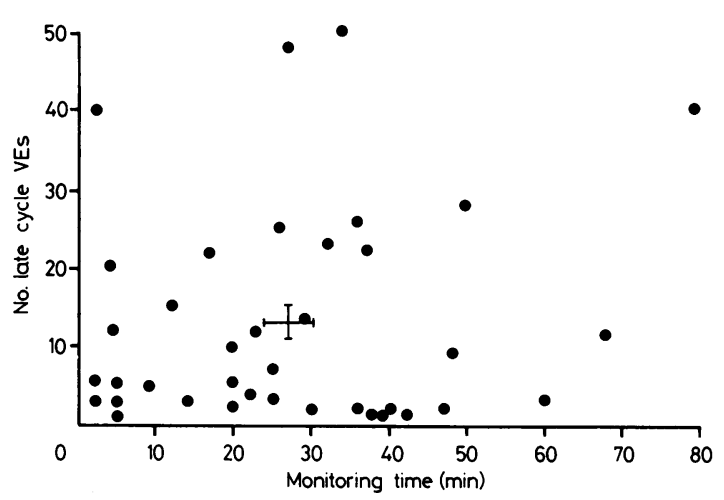

Fig. 2 Frequency distribution of late cycle VEs (ventricular extrasystoles) (38 patients) in relation to monitoring time (mean 13 late cycle ventricular extrasystoles for average monitoring time of 27 minutes).

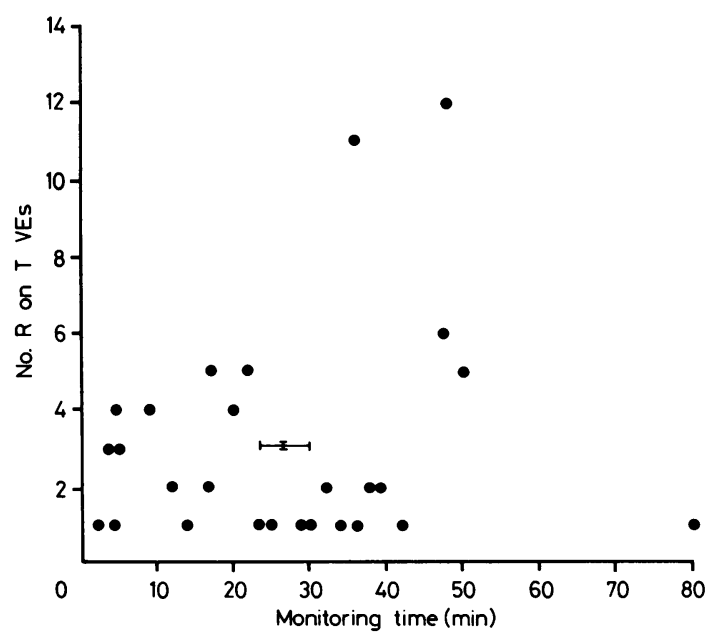

Fig. 3 Frequency distribution of $R$ on $T$ extrasystoles (27 patients) in relation to monitoring time (mean three $R$ on $T$ extrasystoles for average monitoring time of 27 minutes). 
An $\mathrm{R}$ on $\mathrm{T}$ extrasystole initiated ventricular fibrillation in 33 of the 48 patients (Fig. 4). In three patients a late cycle ventricular extrasystole gave rise to ventricular fibrillation, idioventricular rhythm in a further three, and ventricular tachycardia in nine (Fig. 4).

Twenty-nine $(60 \%)$ had an isolated episode of ventricular fibrillation and $19(40 \%)$ had one or more episodes of ventricular fibrillation outside hospital after correction of the initial episode. Thirty-five (73\%) patients survived to leave hospital.

\section{Discussion}

Sudden cardiac death is the leading cause of death among men aged 20 to 64 years. ${ }^{2}$ Though ventricular fibrillation is the major cause of sudden death, ${ }^{3-5}$ many aspects of sudden death still remain unsolved. Factors initiating ventricular fibrillation are largely unknown since this arrhythmia rarely develops in the community in the presence of continuous electrocardiographic monitoring. ${ }^{67}$ Many sudden deaths are instantaneous and therefore do not allow time for electrocardiographic monitoring.

The significance of "warning" or premonitory arrhythmias which precede the development of ven- tricular fibrillation still gives rise to major debate $\frac{\mathscr{N}}{7}$ Generally accepted criteria for "warning arrhyth? mias" include ventricular extrasystoles falling into one of the four following categories: $R$ on $T,>5 \frac{5}{f}$ minute, multifocal, coupled or in salvos. ${ }^{8}$ In experimental and clinical studies $R$ on $T$ extrasystoles have been reported with varying frequency. A study in baboons showed that $69 \%$ who developed ventricular fibrillation within the first hour of acute myocardiat infarction had $R$ on T extrasystoles. ${ }^{9}$ In one study i $\overline{ }$ was found that of patients admitted within six hours:of the onset of infarction, only $10 \%$ of those whow developed primary ventricular fibrillation had $R$ on $T$ extrasystoles as "warning arrhythmias". ${ }^{10}$ Of those with primary ventricular fibrillation, occurring in thêे majority within 24 hours of the onset of infarction $\vec{E}$ only $25 \%$ had antecedent $R$ on $T$ extrasystoles. ${ }^{11}$ It is of interest that in these two studies 1011 as manis patients had $R$ on $T$ extrasystoles who went on to develop ventricular fibrillation as those who did not

It may well be that the pattern or timing of ven tricular extrasystoles as "warning arrhythmias" is now necessarily crucial to the development of ventricularfibrillation but the capacity of the infarcted ventricle to sustain repetitive beating. ${ }^{12}$ In this study during the premonitory phase late cycle ventricular extrasys
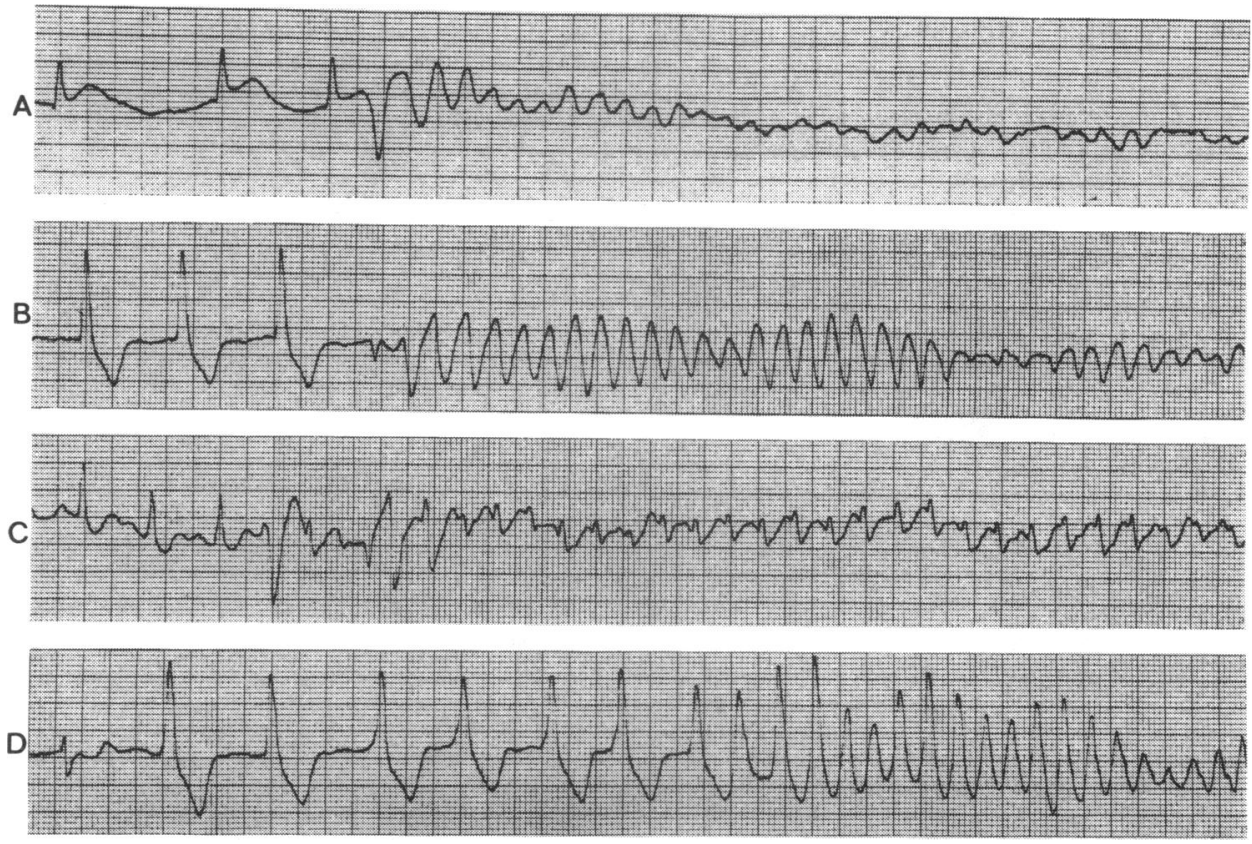

Fig. 4 Initiation of ventricular fibrillation. (A) $R$ on $T$ extrasystole; $(B)$ late cycle extrasystole; $(C)$ ventricular tachycardia/ventricular flutter which degenerated into ventricular fibrillation; (D) idioventricular rhythm with rapid acceleration into ventricular fibrillation. 
toles were the most frequent, occurring in 38 (79\%) patients. $\mathbf{R}$ on $\mathrm{T}$ ventricular extrasystoles were present in $27(56 \%)$ patients. Only an average of three $R$ on $\mathrm{T}$ extrasystoles were recorded, however, during a mean monitoring time of 27 minutes. Multifocal ventricular extrasystoles, consecutive or bigeminal extrasystoles were relatively infrequent. Ventricular extrasystoles occurring with a frequency of $>5$ / minute were rare. In some patients a burst of ventricular extrasystoles immediately preceded the initiation of ventricular fibrillation (Fig. 5). Sustained ventricular tachycardia is rare during the early phase of acute myocardial infarction. ${ }^{113}$ In this study, selfterminating ventricular tachycardia before the development of ventricular fibrillation occurred in three $(6 \%)$ patients.

The time from the development of "warning arrhythmias" until ventricular fibrillation in many cases was very short and this precluded the institution of antiarrhythmic therapy. It therefore calls into question the significance of "warning arrhythmias" in the administration of antiarrhythmic therapy.

Major controversy still surrounds the initiation of ventricular fibrillation. Clinical significance of the vulnerable phase ( $R$ on $T)$ was first emphasised by Smirk and Palmer. ${ }^{14}$ In one study ${ }^{15}$ an $R$ on $T$ extrasystole was the initiating event in $80 \%$ of those who developed primary ventricular fibrillation. In similar studies ${ }^{1011}$ the initiating event was an $R$ on $T$ extrasystole in $45 \%$ and $50 \%$, respectively. Clinical tests and experimental work have shown that ventricular fibrillation may be initiated by an $R$ on $T$ or a late cycle extrasystole. ${ }^{12} 16$ In our study an $R$ on $T$ extrasystole was the most important factor in the initiation of ventricular fibrillation: it was found in $69 \%$ of the patients. A late cycle ventricular extrasystole or idioventricular rhythm rarely initiated ventricular fibrillation. Ventricular fibrillation was initiated by ventricular tachycardia in $19 \%$ of patients. In a study ${ }^{7}$ of seven patients with coronary artery disease who died suddenly during ambulatory monitoring, ventricular tachycardia degenerated into ventricular fibrillation in all seven.

Clinical and experimental studies have shown that ventricular fibrillation may be associated with extremes of heart rate, bradycardia, and tachycardia. ${ }^{11718}$ Sympathetic overactivity most frequently associated with anterior infarction was present in $35 \%$ of patients seen within 30 minutes of the onset of symptoms. ${ }^{1}$ During the movement of patients with acute myocardial infarction, and despite analgesia, a heart rate of $\geqslant 100 /$ minute has been recorded in $45 \% .{ }^{19}$ Increased plasma catecholamine levels have been reported in animals after left anterior descending coronary artery occlusion and in man during the first hour of acute myocardial infarction. ${ }^{20}$ Sympathetic overactivity in association with acute myocardial infarction may cause a fall in ventricular fibrillation

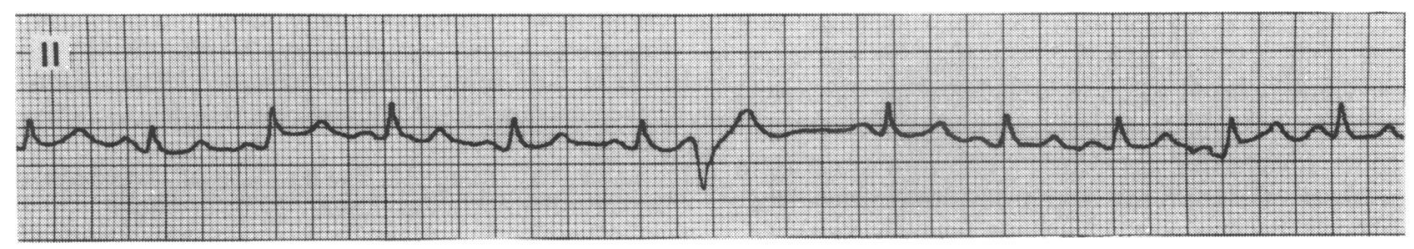

$11 \mathrm{~min}$ later
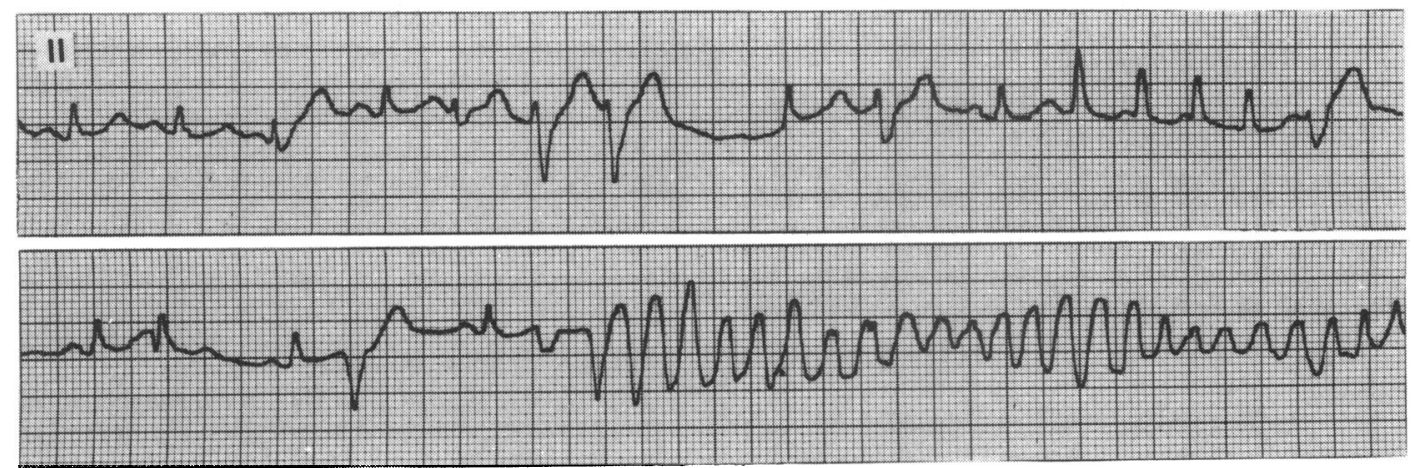

Fig. 5 Top tracing-single $R$ on $T$ extrasystole during out-of-hospital monitoring, heart rate less than $100 /$ minute (lead II ECG). Eleven minutes after top tracing, heart rate increased, burst of late cycle and $R$ on $T$ extrasystoles ending in ventricular fibrillation (second and third tracings continuous). 
threshold, ${ }^{21}$ and since heart rate is a major determinant of myocardial oxygen demand, an increase in the extent of myocardial ischaemic injury. When betablockers are administered in the experimental situation, the incidence of ventricular fibrillation is reduced. ${ }^{22}{ }^{23}$ In this study the heart rhythm immediately preceding the initiation of ventricular fibrillation did not differ significantly from that reported when the patient was seen initially, but there was a significant increase in the heart rate from the time the patient was first seen until that recorded immediately before ventricular fibrillation. This was so even after excluding patients who had received prior treatment with atropine or beta-blocking agents. When ventricular fibrillation occurred during the movement of a patient to hospital there was also a significant increase in heart rate from that recorded initially.

In a study of long and short term survivors from out-of-hospital ventricular fibrillation ${ }^{5}$ it was found that acute myocardial infarction was present in only $39 \%$, and ischaemia without infarction in $34 \%$. In long term survivors of out-of-hospital ventricular fibrillation, myocardial necrosis based on electrocardiographic or enzyme studies was present in $49.5 \%$, and only $17 \%$ had an acute transmural infarction. ${ }^{4}$ In $50.5 \%$ of their patients there was no evidence of myocardial necrosis. Of the group in ventricular fibrillation when the Seattle unit arrived, myocardial necrosis was present in $42 \%$ and only $10 \%$ showed acute transmural infarction. In contrast with those who developed ventricular fibrillation after arrival of the unit, $79 \%$ had evidence of myocardial necrosis, and $50 \%$ showed acute transmural infarction. In our study where ventricular fibrillation occurred after the arrival of the unit, $96 \%$ of short and long term survivors had an acute myocardial infarction. The minimum time from onset of symptoms to the development of ventricular fibrillation was 32 minutes. No patient suffered instantaneous death. It is impossible to say, therefore, that the factors leading to ventricular fibrillation in this group of patients would be identical in those who die instantaneously.

In this study more patients had an anterior than a diaphragmatic infarction. In two studies ${ }^{11} 15$ anterior infarctions dominated and in another study ${ }^{5}$ lesions of the anterior wall dominated, yet in one report ${ }^{24}$ there was a predominance of inferior infarctions. In an earlier study from Belfast, of ventricular fibrillation outside hospital, there was an equal incidence of anterior and inferior infarctions. ${ }^{3}$

\section{References}

1 Pantridge JF, Webb SW, Adgey AAJ, Geddes JS. The first hour after the onset of acute myocardial infarction. In: Yu PN, Goodwin JF, eds. Progress in cardiology. Vol. III. Philadelphia: Lea \& Febiger, 1974: 173-88.
2 Lown B. Sudden cardiac death: the major challenge confronting contemporary cardiology. Am $\mathcal{F}$ Cardiol 1979; $C$. 43: 313-28.

3 Adgey AAJ, Nelson PG, Scott ME, et al. Management of? ventricular fibrillation outside hospital. Lancet 1969; i:? 1169-71.

4 Baum RS, Alvarez H III, Cobb LA. Survival after resus $\overline{\bar{n}}$. citation from out-of-hospital ventricular fibrillation. $C i r$ 的 culation 1974; 50: 1231-5.

5 Liberthson RR, Nagel EL, Hirschman JC, Nussenfeldœ SR, Blackbourne BD, Davis JH. Pathophysiologic observations in prehospital ventricular fibrillation andsudden cardiac death. Circulation 1974; 49: 790-8.

6 Gradman AH, Bell PA, DeBusk RF. Sudden death dur. ing ambulatory monitoring. Circulation 1977; 55: 210 1.

7 Klein RC, Vera Z, Mason DT, DeMaria AN, Awan NA Amsterdam EA. Ambulatory Holter monitor documen tation of ventricular tachyarrhythmias as mechanism of sudden death in patients with coronary artery disease (abstract). Clin Res 1979; 27: 7A.

8 DeSanctis RW, Block P, Hutter AM Jr. Tachyarrhyth mias in myocardial infarction. Circulation 1972; 45? 681-702.

9 Bruyneel KJJ, Opie LH. The value of warning arrhyth mias in the prediction of ventricular fibrillation within one hour of coronary occlusion. Experimental studies info the baboon. Am Heart $\mathcal{f}$ 1973; 86: 373-84.

10 Lie KI, Wellens HJJ, Downar E, Durrer D. Observao tions on patients with primary ventricular fibrillation complicating acute myocardial infarction. Circulation $\bar{b}$ 1975; 52: 755-9.

11 El-Sherif N, Myerburg RJ, Scherlag BJ, et al. Elec $\stackrel{2}{\mathrm{D}}$ trocardiographic antecedents of primary ventricular fibrillation. Value of the R-on-T phenomenon in myocardial infarction. Br Heart $\mathcal{F}$ 1976; 38: 415-22.

12 Engel TR, Meister SG, Frankl WS. The "R-on-T'? phenomenon. An update and critical review. Ann Intern. Med 1978; 88: 221-5.

13 Adgey AAJ, Allen JD, Geddes JS, et al. Acute phase of myocardial infarction. Lancet 1971; ii: 501-4.

14 Smirk FH, Palmer DG. A myocardial syndrome: with particular reference to the occurrence of sudden death and of premature systoles interrupting antecedent $\mathrm{T}$ waves. Am f Cardiol 1960; 6: 620-9.

15 Dhurandhar RW, MacMillan RL, Brown KWG. Prim을 ary ventricular fibrillation complicating acute myocardia infarction. Am $\mathcal{F}$ Cardiol 1971; 27: 347-51.

16 Williams DO, Scherlag BJ, Hope RR, El-Sherif N, Laz zara $R$. The pathophysiology of malignant ventriculaor arrhythmias during acute myocardial ischemia. Circula tion 1974; 50: 1163-72.

17 Chadda KD, Banka VS, Helfant RH. Rate dependen $\tilde{H}$ ventricular ectopia following acute coronary occlusion The concept of an optimal antiarrhythmic heart rateo Circulation 1974; 49: 654-8.

18 Scherlag BJ, Helfant RH, Haft JI, Damato AN. Elece trophysiology underlying ventricular arrhythmias due to coronary ligation. Am F Physiol 1970; 219: 1665-71. $\overline{0}$

19 Mulholland HC, Pantridge JF. Heart-rate changes dure ing movement of patients with acute myocardial infarç tion. Lancet 1974; i: 1244-7. 
20 Strange RC, Vetter N, Rowe MJ, Oliver MF. Plasma cyclic AMP and total catecholamines during acute myocardial infarction in man. Eur $\mathcal{F}$ Clin Invest 1974; 4: 115-9.

21 Kliks BR, Burgess MJ, Abildskov JA. Influence of sympathetic tone on ventricular fibrillation threshold during experimental coronary occlusion. Am $\mathcal{F}$ Cardiol 1975; 36: 45-9.

22 Khan MI, Hamilton JT, Manning GW. Protective effect of beta-adrenoceptor blockade in experimental coronary occlusion in conscious dogs. Am $\mathcal{F}$ Cardiol 1972; 30: 832-7.
23 Khan MI, Hamilton JT, Manning GW. Early arrhythmias following experimental coronary occlusion in conscious dogs and their modifications by beta-adrenoceptor blocking drugs. Am Heart $\mathcal{F}$ 1973; 86: 347-58.

24 Lie KI, Wellens HJ, Durrer D. Characteristics and predictability of primary ventricular fibrillation. Eur $\mathfrak{f}$ Cardiol 1974; 1: 379-84.

Requests for reprints to Dr A A J Adgey, Regional Medical Cardiology Centre, Royal Victoria Hospital, Grosvenor Road, Belfast BT12 6BA, Northern Ireland. 